\title{
A MIXED-METHODS, COMMUNITY-BASED STUDY TO IDENTIFY PREDICTORS OF DEPRESSION IN LATINO ADOLESCENTS BY GENDER
}

\section{SILVIA M. BIGATTI, VIRNA DIAZ, KATRINA K. CONRAD, MICHELLE RAMIREZ, AND TESS D. WEATHERS}

\section{ABSTRACT}

Latino adolescent depressive symptoms are a growing national problem. In this mixed-method, community-based participatory research study we quantitatively examined predictors of depression known to impact adolescent mental health that are amenable to interventions. Concurrently, we qualitatively assessed parents' perceptions of mental health problems in children, their causes and potential solutions. The data from parents $(\mathrm{n}=108)$ was obtained in focus groups led in Spanish, and the data from the majority of adolescents $(n=86)$ was obtained in English language surveys. Among the adolescents there was an even representation of males (47.7\%) and females $(52.3 \%), \mathrm{M}_{\mathrm{age}}=15.24(\mathrm{SD}=1.97)$. Nearly half $(47.7 \%)$ of the adolescents were experiencing minor depression and one in ten (10.5\%) were experiencing major depression according to their scores on the 9item Patient Health Questionnaire (PHQ-9).

Adolescent participants reported low acculturative stress, average social support, and high mastery, as well as highly functional families. Males reported higher self-mastery than females and lower acculturative stress. Predictors of depression differed by gender. For males, self-mastery predicted lower depressive symptoms; for females acculturative stress predicted higher depressive symptoms. The focus groups with parents supported and expanded quantitative findings. The parents demonstrated a keen awareness of depression in teens and their own contributions to the problem, including their efforts to maintain their culture of origin, which may contribute to their teens' acculturative stress. Parents also reported difficulties knowing what steps to take and finding resources. The additional dimension of parental voice is often missing from studies of adolescents, and here it clarified many of the issues identified in the teens. These findings suggest the need to focus on mental health in this population, potentially developing differential interventions by gender and taking a family systems approach.

Keyword: Depression, acculturative stress, selfmastery, Latino Adolescents, gender.

\section{INTRODUCTION}

In the United States, more than four-in-ten Latinos can be found in 10 metropolitan areas, six of which are located in California and Texas (Brown \& Lopez, 2013). However, the Latino population has been establishing roots in other communities across the country. From the year 2000 to 2011, among the 60 
metropolitan areas with the largest growth in Latino population, Indianapolis, Indiana saw the highest increase at $197 \%$ - this represents a growth from 33,290 to 84,466 in that decade alone (Brown \& Lopez, 2013). Many of those that arrived in Indiana during those years settled in the state and started families. Among the then 426,00o individuals who identified as Latino in the whole state, $32 \%$ were foreign born, and $76 \%$ were of Mexican origin, $8 \%$ Puerto Rican, $6 \%$ Central American, 2\% Cuban/Dominican, 2\% South American, and $6 \%$ Other (Strange, 2013). The average age of the foreign born subgroup was 36 , and the average age of the US born was 16 (Pew Research Center, 2014). These Latino parents are now facing the challenges of raising adolescents in a culture and environment different from their own, and far from extended family support. In these newer migration destinations for the Latino population, Latino parents cannot depend on the resources and connections present in communities with long-established Latino populations, and are more likely to have unmet needs, especially parenting support. This is a problem given the mental health needs identified in Indiana teens, and Latino teens in particular.

Specifically, compared to the US as a whole (11.9\%), a higher percentage of Indiana youth (14.6\%) experienced a major depressive episode in 2014 (SAMHSA, 2015). A 2015 report from the Youth Risk Behavior Survey (YRBS) identified higher rates of suicide attempts in Latino youth (15.5\%) in the previous year compared to their non-Hispanic White peers $(8.7 \%)(C D C, 2015)$. These data point to a serious problem for Latino youth, one that VD, the community partner, and SB and her team, the academic partners, decided to explore jointly given their shared interests in the wellbeing of this population.

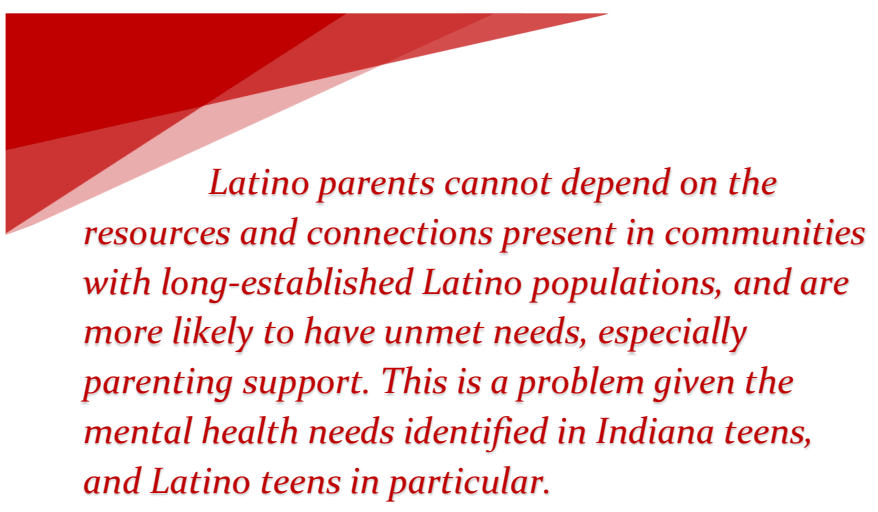

We sought to identify predictors of depression in Latino youth, as a first step in developing community-based prevention programs. We examined intrapersonal factors (acculturative stress, mastery) and interpersonal factors (social support, family functioning) known to impact adolescent mental health in general and to be amenable to interventions. Acculturative stress may contribute to depressive symptoms among the teens who are themselves immigrants or have immigrant parents (Lawton \& Gerdes, 2014; Lorenzo-Blanco, Unger, BaezcondeGarbanai, Ritt-Olson, \& Soto, 2012; Roche \& Kuperminc, 2012). Acculturative stress is the stress experienced as individuals go through the process of acculturation and adjustment between their native cultural values and customs and the mainstream American customs and cultural values (Lawton \& Gerdes, 2014; Stein, Gonzalez \& Huq, 2012). For these teens, acculturation to the US may happen more quickly than for their parents, potentially causing problems at home (Cox Jr., Zapata Roblyer, Merten, Shreffler \& Schwerdtfeger, 2013; Lawton \& Gerdes, 2014). 
Teens experiencing acculturative stress may be at risk for depression; yet a well-functioning family, as well as self-mastery and social support, may be protective (Kuperminc, Wilkins, Roche \& AlvarezJimenez, 2009; Lorenzo-Blanco et al., 2012). A family that is strong and cohesive will not only protect against stress and depression, but may be less likely to contribute to stress, especially acculturative stress (Dillon, De La Rosa \& Ibañez, 2013). Teens high in selfmastery are more likely to see stressors as temporary events that they will be able to overcome, and are less likely to experience depression. Social support is instrumental to mental health and protective against depression for youth (Rueger, Malecki, Pyun, Aycock \& Coyle, 2016). Low peer, family and school social support are associated with suicidal ideation and attempts in youth (Miller, Esposito-Smythers \& Leichtweis, 2015).

During adolescence especially, females face higher risk for depressive symptoms than males overall (Mojtabai, Olfson \& Han, 2016). Moreover, adolescent Latina females are more likely to suffer from more severe depressive symptoms than their male counterparts, and are more likely to attempt suicide than any other race and ethnicity (Lorenzo-Blanco et al., 2012), thus gender is an important factor to investigate when considering the mental health outcomes of Latino adolescents (Lorenzo-Blanco et al., 2012).

Therefore, in the present study we examined depressive symptoms among Latino teens in an emerging Latino community, focusing on gender differences, acculturative stress, and protective factors, such as family support. We assessed parents because parental perceptions are key to understanding youth mental health issues (Miller, Esposito-Smythers \& Leichtweis, 2015).

\section{METHODS}

\section{COMMUNITY-BASED PARTICIPATORY}

\section{RESEARCH (CBPR)}

The present study was conducted using a Community-Based Participatory Research (CBPR) approach. CBPR "equitably involves all partners ... with a research topic of importance to the community with the aim of combining knowledge and action for social change to improve community health and eliminate health disparities" (Wallerstein \& Duran, 2010, p. S40).

We employed several key principles of community-based research as described by Israel and colleagues (1998). First, we developed the study collaboratively after discussing the challenges that Indiana Latinos face. Once the study was developed, the entire team built upon the strengths of the community partner in terms of knowledge about the community and their existing cadre of community contacts for recruitment purposes. Throughout the duration of the study, both the community partner and the academic partners were actively engaged and included in all phases of research. Both the community partner and academic partners conceptualized and wrote this manuscript.

The study described here was the first collaboration of this community-university partnership. The academic partners (SB, KC \& TW) shared with IMHC their interest in focusing on Latino youth mental health after exploring statistics regarding this health disparity. The Indiana Minority Health 
Coalition (IMHC) facilitated the connection with the Latino Health Organization. Once we were introduced, we began to jointly explore the literature and decide on the focus and methodology of the study, which was funded by IMHC.

\section{DESIGN}

The study employed a concurrent mixedmethods research design with different participants for each method of data collection. Quantitative data were obtained from adolescent participants via several survey instruments, while at the same time complementary qualitative data were collected from parents in focus group sessions. These adolescent and parent study sessions were held simultaneously. The academic partners and the community partner were involved in data collection.

\section{RECRUITMENT}

Following university IRB approval, the community partner initiated recruitment. Selfidentified Latino adolescents and their parent(s) living in the Indianapolis metropolitan area were eligible to participate. Phone calls were made and/or letters were sent to clients of the community partner with adolescent children, requesting that only one adolescent per family participate, chosen by the parent. The community partner also sent flyers and letters to community centers or churches where she has pre-existing ties and approval. Families who learned about the study through the flyers or letters and were interested in participating called the community partner and signed up for the study. Eight meetings were planned and held in community locations in two counties, one urban, one suburban, over a time period of four months. Each session lasted 6o-9o minutes.

\section{CONSENT AND ASSENT}

The study was thoroughly explained in Spanish to parents and adolescents. Opportunity was given to all potential participants to ask questions. A study information sheet was available for their convenience. It was made clear to both parents and adolescents that the responses that each gave would be treated as confidential and anonymous by the study team. A process of implied or passive assent/consent was employed; attendees who did not wish to participate following the study explanation were given the opportunity to leave the event. At this point, parents and adolescents were separated into different rooms in the facility to conduct the separate assessments. To participate, at least one parent and one teen per family had to voluntarily consent to be part of the study; however, in some cases both parents participated in the focus groups.

\section{STUDY PROCEDURES}

\section{Adolescents}

No identifying information was collected from any participant, primarily to encourage adolescents to answer honestly without worry that their parents may discover how they answered. The researchers offered to each adolescent participant 1) written surveys in English or Spanish, whichever they preferred, or 2) the option to have the survey questions read out loud (privately) in the case that there were any participants who had trouble reading or writing. Eight ( 4 girls and 4 boys) completed surveys in Spanish; none asked to have the surveys read to them. They were informed that they could ask any questions if needed, and also 
stop if they felt tired or uncomfortable. On average, it took approximately 6o-75 minutes to complete the questionnaires and participants received a $\$ 10$ gift card for their time. $\mathrm{KC}$ managed the adolescent data collection with the help of research assistants. Parents

At the same time the adolescents were completing their questionnaires, parents participated in a focus group session. More than one parent per child could, and sometimes did, participate. Members of the group were asked a variety of questions in Spanish to explore their perspectives on the overall study theme of stress and sadness among Latino adolescents.

Parents were asked to think of adolescents in general, including their own children and their children's friends, to reduce fear of personal disclosure. Questions included:

1) How much stress do you think adolescents are under, and why?

2) What does this stress look like in adolescents? (eg.: he/she cries frequently, sleeps too much, is not hungry, does not socialize)

3) What do you think is causing stress in adolescents?

4) What might parents do that causes more stress to their adolescent child?

5) What can parents do to help their adolescent child deal with stress?

6) How sad do you think adolescents are in general, and why?
7) How worried are you about the behavior of adolescents in their daily life environment, and why?

8) What specifically are adolescent children doing that worries you, and why?

9) How much do you think the new culture is related to the stress in Latino adolescent children, and why?

These focus group discussions were co-led in Spanish-only by SB and VD. The choice of Spanishonly was based on the community partner's knowledge that adults in the Latino community she serves are mostly limited English proficient. She informed parents of the language of the focus groups when she recruited them. Mean number of parents per focus group was 13.5 (median $=13$ ), with a low of 11 parents in one of the groups and a high of 15 parents in two of the groups. Parents were told that they could stop participation at any time if they felt uncomfortable, and none were required to answer any of the questions. No identifying information or demographics were collected from parents on advice from the community partner (VD), to encourage participation, increase their comfort level with participation, and confirm the anonymous nature of participation. With participants' consent, the discussions were audiorecorded for subsequent transcription. Upon conclusion of each focus group, each parent received a $\$ 10$ gift card as a token for participation.

\section{MEASURES (FOR ADOLESCENTS)}

Demographics. The demographic form included age, gender, immigrant status (immigrant, first, or second generation), language most spoken 
with friends, language most spoken with family, and proportion of Latino friends.

Depression. To measure depression levels, the PHQ-9 (Kroenke \& Spitzer, 2002) was utilized. The PHQ-9 is a 9-item instrument that measures different manifestations of depressive symptoms and has been validated in several languages, including Spanish. The answers selected (on a 4-point scale) range from $o=$ Not At All to 3 = Nearly Every Day. Sample items include: "little interest or pleasure in doing things," "feeling down, depressed, or hopeless," and "feeling tired or having little energy.” Scores range from o to 27 when computing raw scores. There are also cut-offs for depressive severity diagnostic categories. Scores from o to 4 suggest minimal or no depression, 5 to 9 suggest mild depression, 10 to 14 suggest moderate depression, 15 to 19 suggest moderately severe depression, and scores from 20 to 27 suggest severe depression. In the literature, the PHQ-9 has been shown to be reliable. In a study of Hispanic American women, the reliability was $\alpha=.84$ in the sample who chose the English version and $\alpha=.85$ in the sample who chose the Spanish version (Merz, Malcarne, Roesch, Riley, \& Sadler, 2011). Reliability in the present sample was $\alpha=$ .87 for the English version.

Acculturative Stress. The Social, Attitudinal, Familial, and Environmental (SAFE) scale (Mena, Padilla \& Maldonado, 1987) was utilized to measure acculturative stress. The SAFE scale is a 24-item survey rated on a 5 -point scale from $1=$ Not Stressful to $5=$ Extremely Stressful. Sample items include: "It bothers me that family members I am close to do not understand my new values," "It bothers me that I cannot be with my extended family," and "I don't feel at home in this country." A 'does not apply' response option was also available, with a score of o. Thus, possible scores ranged from o to 120. In the literature, the SAFE scale has been shown to be reliable with $\alpha=$ .89 (Mena et al, 1987). VD, SB and MR translated the SAFE into Spanish. Reliability in the present sample was $\alpha=.90$ for the English version.

Social Support. The Child and Adolescent Social Support Scale (CASSS) (Malecki, Demaray \& Elliot, 200o) was utilized to measure perceived support from family and school. The CASSS consists of 60 items that may be broken down into five subscales of 12 items rated on a 6 -point scale from $1=$ Never to $6=$ Always. Subscales measure perceived social support from parents, teachers, class, school, and close friends. The full scale ranged from 6o to 360 , with each subscale ranging from 12 to 72 . Sample items include: "My close friends understand my feelings," "My teachers make sure I have what I need for school," and "My parents show they are proud of me." In the literature, the CASSS has been shown to be reliable with $\alpha=.96$ (Malecki \& Demaray, 2003). We used the full scale in the present study to preserve statistical power and to capture the totality of available support from all sources. VD, SB and MR translated the CASSS into Spanish. Reliability in the present sample was $\alpha=$ .97 for the English version.

Self-Mastery. In order to measure self-mastery, Pearlin and Schooler's (1978) Self-Mastery Scale was utilized. The Self-Mastery Scale is 7 total items that are scored on a 5-point scale from $1=$ Strongly Disagree to 5 = Strongly Agree. Scores had possible ranges from 7 to 35. Sample items include: "I have little control over the things that happen to me," "I often feel helpless in 
dealing with the problems of my life," and "What happens to me in the future mostly depends on me." In the literature, the Self-Mastery Scale has been shown to be reliable with $\alpha=.74-.75$ (Scheier, Carver, \& Bridges, 1994). Reliability for the Spanish version has been reported at $\alpha=.74$ (Rini, Wadhwa \& Sandman, 1999). Reliability in the present sample was $\alpha=.80$ for the English version.

Family Functioning. The Family APGAR (Smilkstein, 1978) was utilized to measure perceived family functioning. The Family APGAR consists of 5 items that are scored on a 5-point scale ranging from 1 $=$ Always to $5=$ Never. Scores had possible ranges from o to 10. Sample items include: "I am satisfied with the way my family and I share time together," "I am satisfied that my family accepts and supports my wishes to take on new activities or directions," and "I am satisfied that I can turn to my family for help when something is troubling me." In the literature, the Family APGAR has shown to be reliable with $\alpha=.77$ (Gómez \& Ponce, 2010). The Spanish version has shown reliability of $\alpha=.84$ (Bellon-Saameno, DelgadoSanchez, Luna del Castillo, \& Lardelli-Claret, 1996). Reliability in the present sample was $\alpha=.86$ for the English version.

\section{DATA ANALYSIS PLAN}

After each collection session, surveys were taken back to the academic team's office where the results were securely entered into statistical software (IBM ${ }^{\circledR}$ SPSS $^{\circledast}$ Statistics). Our first step was to describe the sample and the variables of interest, and determine relations among them. For this purpose, descriptive and bivariate analyses were completed for each collected measure. Our second step was to determine what factors may explain depression and whether these differed by gender. For this purpose, regression analyses were used to examine predictors of depression and multivariate analyses of variance to compare males and females. A power-analysis for the regressions with $\mathrm{G}^{*}$ Power, alpha $=.05$, power $=.8 \mathrm{o}$, four predictors indicated a needed sample size of 84 (Faul, Erdfelder, Buchner \& Lang, 2009).

Focus groups were analyzed following a thematic approach (Boyatzis, 1998). Audiotaped focus group discussions were transcribed and translated verbatim by one of the authors (MR). Independently, MR and SB identified themes, and then met to discuss the themes and reach agreement on the set of themes to be used. They each then coded the transcripts according to the themes. Once the transcripts were coded, VD and KC verified the results. Crosscomparisons of focus group results for consistency with findings from adolescent surveys are detailed below.

\section{RESULTS}

\section{DEMOGRAPHICS}

Our study sample consisted of 86 Latino adolescents $\left(\mathrm{M}_{\mathrm{age}}=15.24, \mathrm{SD}=1.97\right)$, and 108 parents. Demographics were not collected from parents participating in focus groups at the recommendation of the community partner to increase participation and facilitate disclosure. The responses from adolescents show that all but 3 parents were foreign-born (see Table 1). Among the adolescent participants, there was even representation of males $(47.7 \%)$ and females (52.3\%). See Table 1 for additional adolescent demographic information by gender; Chi-square 
analyses showed no differences by gender in any of these demographic variables.

Proxy measures of acculturation among the demographics, such as language spoken outside the home (English was between $82.2 \%$ and $91.1 \%$ of the sample in the present study) and choice of friends (Mostly or All Hispanic, between 57.7\% and 68.3\%), suggest that overall, this sample of adolescents was already acculturated. This is noteworthy given that most of the adolescents were foreign-born $(68.9 \%$ for females, and $56.1 \%$ for males).

Table 1: Demographic information by gender

\begin{tabular}{|c|c|c|}
\hline & Females $(\mathrm{n}=45)$ & Males $(n=41)$ \\
\hline Age & Mean $=15.20(\mathrm{SD}=2.19)$ & Mean $=15.29(\mathrm{SD}=1.72)$ \\
\hline \multicolumn{3}{|l|}{${\text { Immigrant status }{ }^{1}}$} \\
\hline I was born outside US & $68.9 \%$ & $56.1 \%$ \\
\hline I was born inside US & $28.9 \%$ & $39 \%$ \\
\hline $\begin{array}{l}\text { My parents and I were } \\
\text { born in US }\end{array}$ & $2.2 \%$ & $4.9 \%$ \\
\hline \multicolumn{3}{|l|}{ Ethnicity of friends ${ }^{2}$} \\
\hline Mostly Hispanic & $53 \cdot 3 \%$ & $61 \%$ \\
\hline Mostly not Hispanic & $35.6 \%$ & $24.4 \%$ \\
\hline All Hispanic & $4.4 \%$ & $7.3 \%$ \\
\hline All not Hispanic & $6.7 \%$ & $7.3 \%$ \\
\hline \multicolumn{3}{|l|}{ Language for survey } \\
\hline English & $91.1 \%$ & $90.2 \%$ \\
\hline Spanish & $8.9 \%$ & $9.8 \%$ \\
\hline \multicolumn{3}{|l|}{ Language with friends } \\
\hline English & $82.2 \%$ & $90.2 \%$ \\
\hline Spanish & $17.8 \%$ & $9.8 \%$ \\
\hline \multicolumn{3}{|l|}{ Language with family } \\
\hline English & $4.4 \%$ & $4.9 \%$ \\
\hline Spanish & $95.6 \%$ & $95.1 \%$ \\
\hline
\end{tabular}

Notes:

${ }^{1}$ Coded as born in US vs. Not for analyses

${ }^{2}$ Coded as all or most Hispanic or Not for analyses
No statistically significant differences by gender on any of these variables.

\section{DESCRIPTIVE AND BIVARIATE ANALYSIS}

Means and standard deviations for study variables by gender can be found in Table 2 . When examining cut-off scores for the PHQ-9, nearly half (47.7\%) were experiencing minor depression and 10.5\% were experiencing major depression. As a group, participants reported low acculturative stress, average social support and high mastery; they also reported highly functional families. Boys and girls differed in self-mastery, $\mathrm{F}(1,84)=6.89, p=.011$, with boys scoring higher than girls. They also differed in acculturative stress, $\mathrm{F}(1,84)=6.68, p=.010$, with girls scoring higher than boys.

Table 2: Correlations and Means (SD) among Study Variables by Gender

\section{FEMALES}

\begin{tabular}{|l|c|c|c|c|c|}
\hline & PHQ-9 & SAFE & CASS & MAST & APGAR \\
\hline $\begin{array}{l}\text { PHQ-9 Depression } \\
\text { (raw score) }\end{array}$ & $\bullet$ & $.679^{* *}$ & $-.306^{*}$ & $-.302^{*}$ & -.268 \\
\hline $\begin{array}{l}\text { SAFE - Acculturative } \\
\text { Stress }\end{array}$ & & $\bullet$ & -.281 & $-.459^{* *}$ & $-.399^{* *}$ \\
\hline $\begin{array}{l}\text { CASSSS - Social } \\
\text { Support }\end{array}$ & & & $\bullet$ & .069 & .247 \\
\hline MAS - Self-Mastery & & & & & .061 \\
\hline $\begin{array}{l}\text { APGA - Family } \\
\text { Functioning }\end{array}$ & & & & & $\bullet$ \\
\hline Mean & 5.48 & 20.28 & 20.54 & 4.68 & 2.32 \\
\hline SD & 0 & 2 & 71 & 14 & 1 \\
\hline Minimum & 25 & 80 & 180 & 32 & 10 \\
\hline Maximum & & & & & \\
\hline
\end{tabular}




\section{MALES}

\begin{tabular}{|c|c|c|c|c|c|}
\hline & $\begin{array}{l}\text { PHQ- } \\
9\end{array}$ & SAFE & CASS & MAST & APGAR \\
\hline $\begin{array}{l}\text { PHQ-9 Depression } \\
\text { (raw score) }\end{array}$ & $\checkmark$ & $.485^{* *}$ & $-.430^{* *}$ & $\begin{array}{c}- \\
.646^{* *}\end{array}$ & $-.498^{* *}$ \\
\hline $\begin{array}{l}\text { SAFE - } \\
\text { Acculturative Stress }\end{array}$ & & $\bullet$ & -.250 & $-.671^{* *}$ & -.273 \\
\hline $\begin{array}{l}\text { CASSSS - Social } \\
\text { Support }\end{array}$ & & & 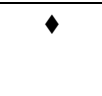 & $.416^{* *}$ & $.697^{* *}$ \\
\hline MAS - Self-Mastery & & & & $\bullet$ & $.397^{*}$ \\
\hline $\begin{array}{l}\text { APGA - Family } \\
\text { Functioning }\end{array}$ & & & & & $\diamond$ \\
\hline Mean & 5.29 & 27.51 & 123.01 & 27.07 & 7.29 \\
\hline SD & 5.91 & 14.07 & 22.66 & 5.27 & 3.08 \\
\hline Minimum & o & 4 & 60 & 16 & o \\
\hline Maximum & 21 & 61 & 183 & 35 & 10 \\
\hline
\end{tabular}

Note. ${ }^{*} p<.05 ;{ }^{* *} p<.01$

Statistically significantly different means by gender: self-mastery $(\mathrm{p}=$ .o11) and acculturative stress $(\mathrm{p}=.010)$

Pearson correlations among variables are shown in Table 2, separated by gender. In general, correlations were low to moderate for all variables; however, it is noteworthy that correlations among some variables differed in strength by gender. In order to determine whether the differences in correlations were statistically significant, we used Fisher r-to-z transformation. With this approach we found that the correlations between depression and self-mastery were statistically significantly different by gender $(z=2.04, p$ $=.04$ ), as were the correlations between social support and family functioning $(z=-2.72, p=.007)$.

We also examined demographic variables and their relationship to depression. Age was moderately correlated to depression $(r=.32, p=.003)$; closer examination showed scores in depression ranged from a low of $\mathrm{M}=3.44(\mathrm{SD}=1.02)$ at age 12 , to a high of $\mathrm{M}=$ $12.78(\mathrm{SD}=2.59)$ at age 18 , with fluctuating scores across ages. One-way analyses of variance examined differences in depression by gender, immigration status, and ethnicity of friends. Females reported higher scores in depression than males, $F(1,84)=4.172$, $p=.044$. When we examined males and females by severity of depression based on cut-off scores, females were more likely to be at least minimally depressed than males (73.3\% compared to $41.5 \%$; see Table 3 ). There was no difference in PHQ-9 scores between those born in the US and those born outside the US, nor among those whose friends are mostly or all Hispanic or not $(p>.05)$.

Table 3: PHQ-9 Depression Diagnostic Categories by Gender

\begin{tabular}{|l|c|c|}
\hline \multicolumn{1}{|c|}{ Diagnostic Category } & $\begin{array}{c}\text { Female } \\
\mathrm{n}(\%)\end{array}$ & $\begin{array}{c}\text { Male } \\
\mathrm{n}(\%)\end{array}$ \\
\hline Minimal or no depression (score ranges 0-4) & $12(26.7)$ & $24(58.5)$ \\
\hline Mild depression (score ranges 5-9) & $19(42.2)$ & $8(19.5)$ \\
\hline Moderate depression (score ranges 10-14) & $9(20)$ & $5(12.2)$ \\
\hline Moderately severe (score ranges 15-19) & $4(8.9)$ & $2(4.9)$ \\
\hline Severe depression (score ranges 20-27) & $1(2.2)$ & $2(4.9)$ \\
\hline
\end{tabular}

\section{MAIN QUANTITATIVE ANALYSES}

In order to examine the combined relationship of all predictor variables with depression, we ran regression analyses. Separate regression analyses were run examining predictors of depression for each gender to examine strengths of association and prediction values between groups. For both males and females, depressive symptoms (total PHQ-9 scores), the dependent variable, were regressed onto the various predictors: acculturative stress (SAFE), social support (CASSS Total Scores), self-mastery, and family functioning (Family APGAR). The regression for females was statistically significant, $\mathrm{F}(4,40)=9.09, p<$ 
.o1. The full model explained a total of $42.4 \%$ of the variance in depression. Only acculturative stress showed enough strength to enter the regression equation $(\beta=.656, p<.001)$, with higher stress predicting higher depression. The regression for males was also statistically significant, $\mathrm{F}(4,36)=8.69, p<$.o1. The full model explained a total of $49.1 \%$ of the variance in depression. Only self-mastery showed enough strength to enter the regression equation $(\beta=-$ $.467, p=.010$ ), with lower mastery predicting higher depression. These findings suggest that although all predictors as a whole played a role in male and female depression, there was one specific variable in each group (stress for females and self-mastery for males) that stood out.

\section{QUALITATIVE FINDINGS FROM PARENT}

\section{FOCUS GROUP}

Five major themes emerged from the eight focus group with parents $(n=108)$. These were:

1) Parents' awareness and worry about depression in their children

2) Parents' awareness that their own behaviors and emotions impact their children

3) Parents' lack of resources to deal with stress in the family

4) Minority/immigrant status is both a cause of depression and a barrier to solutions

5) Conflicts between parents and teens are caused by cultural adaptation differences.

\section{THEME 1:}

This first theme addressed the focus of the present study, i.e. the depressive symptoms in Latino teens. Parents were asked several questions about this topic, and were informed that this was the focus of the study. It is therefore not surprising that describing teens' depressive symptoms was a major component of parent responses. The teens' externalizing and internalizing behavior made it clear to parents that something problematic was happening. Some of the comments of parents that indicated problematic changes in behavior included:

"They do not want to talk, so one can tell that something is going on whether it be in school, with friends, at home."

"She was so depressed she would go into the closet and cover herself with a blanket, and that scared me."

"They get into a bad mood. They also demonstrate it with anger...you can tell by their facial expressions...it worries me a lot.” “...my son will isolate himself, lock himself in his room-he does not want to come out." "With the story of the girl [the cutting], I wanted to mention it to my children because I worry a lot. ..."

\section{THEME 2:}

Throughout the focus group meetings, parents showed insight into their own contributions to their children's mental health. These were demonstrated by comments such as:

"We do not always express it, but sometimes the teens will hear us talking about our economic situation, about not being able to pay the rent, to buy food."

"Sometimes there are conflicts between a couple... a lack of money and family problems so they find out and I think that is a cause of great stress for them." 
"We are living in an age where the Latino is persecuted because of the migratory situation. So if the parents are feeling affected, it is to be expected that the adolescents feel it as well." “...sometimes parents make it so that the kids are embarrassed of their background. So we cannot do that because they do not know what or who they are, if they are from here or from there, although they were born here." THEME 3:

However, in spite of recognizing the problem and potential causes, parents often reported feeling helpless to find solutions because of various limitations.

Some of the parents' limitations were financial:

"There are support centers but those are not accessible. ... sometimes our income is not enough to send our teens to a good psychologist or support group."

“...I do not have any other family here to help me take care of them or to give me advice on how to help them. This is difficult for me."

Other times the limitations were related to free time: “...he does not confide because he says, 'You work too much and come home tired and if I tell you my problems..."

"So if they are happy and want to do things but I am not, I am stressed with work and bills, all that, I cannot give them the quality of time that they need. So they become stressed and lock themselves in their room, separate themselves from us."

Mostly parents reported limitations in terms of not knowing what to do:
"As a parent, one feels powerless/helpless. They are at that age where one does not know what to do...we do not want to make them feel worse."

"One does not know how to talk to the girls ... what do I do to take her out of that place to make her understand that I need to be with her and she needs to be with me? I have not been able to achieve that, I do not know how." "They do not know how to communicate with us and we do not know how to be with them. ... I do not know how to go about doing that." THEME 4:

Parents reported that minority/immigrant status was a barrier because of language and cultural differences, interpersonal problems, or legal issues. Examples include:

“...the culture influences them as well because migrating to another country as an adolescent is difficult because they want to go out but it is hard because it is another language."

"[The Latino adolescents] do not identify with the [American teens] because of the language and the school and they do not identify with us because we are in a new country and we do not have our culture, our people."

"My son has talked to me about how he has heard how teens make fun of students that have arrived from other countries so that causes stress.”

“...we do not have documents; they cannot get driver's licenses, all that perturbs them. So all that causes them to constantly think about all 
those stresses and how we are going to pay for college when we do not have a social security number to be able to keep studying..."

\section{THEME 5:}

In this theme parents address the effects on the family of the process of adaptation to the new culture.

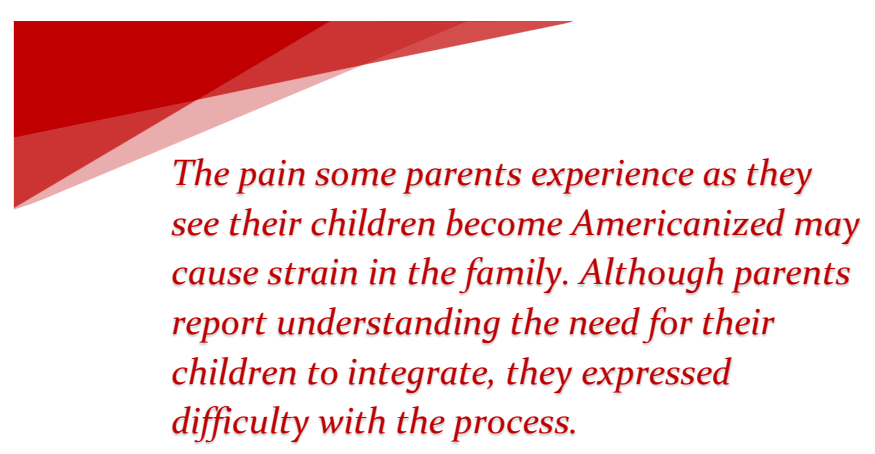

Parents evidenced pain at watching their children adapt and possibly lose their culture of origin, and at times understanding that this may be a necessary step for adaptation. Comments included:

"I impress upon her that she can be American because she was born here but she has our culture. So I have always told her that she has to embrace her culture. That she has Hispanic parents, that she looks Hispanic, that she does not look American, so she has to know that it is her culture, it is her roots and that she should not forget that."

"We as parents are never going to lose our culture. But we also need to understand that they are living in another culture. So for them, many times it is difficult. It provokes stress. So sometimes there is no identification with them because we teach them one thing, but they live in a different culture. And sometimes as parents we close ourselves off; we are also at fault for that."
"Even in the language, they come home talking in

English and one tells them to speak Spanish because it would be terrible to be a Mexican that does not speak Spanish.”

\section{TRIANGULATION OF QUANTITATIVE AND QUALITATIVE FINDINGS}

We found that the focus groups with parents informed and explained the quantitative data from the youth surveys. Our quantitative findings identified various levels of depressive symptoms in the adolescents. These were described by parents as a variety of behaviors, both internalizing and externalizing, in Theme 1.

Furthermore, the quantitative findings suggested that factors within the family contributed to depressive symptoms. Specifically, our assessment of family functioning using the Family APGAR scale, and of social support with the CASSS correlated with depression in boys. The CASSS, but not the Family APGAR, correlated with depression in girls. Theme 2 from the focus groups, where parents recognized that some of their behaviors and emotions contributed to the depressed mood in their children, clarified what sorts of couple and family issues might be leading to the observed correlations.

Theme 4, where parents reported language and cultural differences, interpersonal problems, and legal issues related to minority status, may partially inform why acculturative stress, as measured with the SAFE, correlated with depression among teens. Acculturative stress is the stress experienced as one adapts to a new culture, and the issues identified by parents might be 
interrupting the teens' integration, or making it more challenging.

Theme 5 may be the most interesting and informative for future interventions. In this theme, parents address the effects of acculturation on the family. Parents explain how the process of acculturation and its associated stress (SAFE) relate to family functioning (APGAR). In the quantitative analyses, among females, family functioning correlated with acculturative stress, which was the only statistically significant variable in the regression predicting depression scores in girls. Among boys, family functioning correlated, at similar strength, with mastery, which was the only statistically significant variable predicting depression scores in boys. The pain some parents experience as they see their children become Americanized may cause strain in the family. Although parents report understanding the need for their children to integrate, they expressed difficulty with the process.

\section{DISCUSSION}

The results from the quantitative data gathered from the adolescents are consistent with the findings from other studies. In the present study Latino adolescent females reported higher rates of depression than their male counterparts, which matches extant literature (Lorenzo-Blanco et al., 2012; García, Manongdo \& Ozechowski, 2014). Importantly, we found different predictors of depression among males and females, with girls' depression predicted by higher acculturative stress, and boys' by lower self-mastery. Social support from friends, family and school, and self-mastery correlated with lower depression for girls, but in the presence of higher acculturative stress, did not reach significance in the regression, suggesting that the negative impact of acculturative stress was stronger than the positive impact of social support and self-mastery. For boys, higher social support from friends, family and school, lower acculturative stress, and higher family functioning correlated with depression, but in the presence of self-mastery did not reach significance in the regression, suggesting that the positive impact of self-mastery was stronger than the positive impact of social support and family functioning and the negative impact of acculturative stress.

Latino cultural norms show large differences in how parents raise Latino girls and boys. Traditional Latino cultural norms set expectations that female outside-the-home activities will be more restricted than males' (Lui, 2015; Roche et al, 2018) and that within the home they will perform household activities and be submissive to the rest of the family (Cupito, Stein \& Gonzalez, 2014). These norms differ from the cultural norms in the US, which do not make such marked differences by gender (Raffaelli \& Ontai, 2004). It may not be surprising then that immigrant girls acculturate at a faster rate than their male counterparts, possibly attracted to the greater freedom experienced by adolescent girls in the US (Céspedes \& Huey, 2008; Lorenzo-Blanco et. al, 2012), a freedom that immigrant boys are not denied within their own culture. Unfortunately, this process threatens adolescent girls' mental health (Cupito, Stein \& Gonzalez, 2014). Furthermore, parents who arrive in the US may be struck by the cultural differences in behavior, dress, and freedom for girls. These 
differences may cause them to enforce their own cultural norms even more strictly than they would otherwise. These different cultural norms and rates of acculturation may explain why acculturative stress was the most powerful predictor of depression among the girls.

For boys, who in our sample did not experience as much acculturative stress as girls, the experience of adapting to the new culture may not be as impactful. Instead, the confidence and ability to deal successfully with stressors plays a more salient role. Self-mastery has been consistently found to protect from depression in adolescents (Gilster, 2014; Watkins, Hudson, Caldwell, Siefert \& Jackson, 2011). It is important to note that self-mastery correlated with depressive symptoms at the bivariate level for the girls in our sample as well. Programs that increase self-mastery may help Latino teens, especially first or second generation or those whose parents have yet to acculturate.

Although the focus groups asked parents about teens in general and did not match the parental reports with the children's data, the mixed methods findings suggest other factors that relate to depression as well. Family functioning is invariably impacted by the experience of immigration and adaptation to a new country and culture (Lawton \& Gerdes, 2014). In the focus groups held with the Latino immigrant parents, conflict related to cultural differences they experience with their children was often reported. As parents strived to maintain their cultural identity, their children seemed to strive to assimilate, or at least this was what parents perceived. Parents perceived danger in this, as they saw their culture and its values as sources of strength for themselves and potentially for their children. As the children acculturate, they may not readily adopt, or may even reject, family traditional cultural values, causing even more family conflict and less family cohesion (Lorenzo-Blanco et al., 2012; Lui, 2015). Efforts to solidify family relations in Latino families may reduce family conflict. Familismo, a Latino cultural value of loyalty, respect and obedience to family, is protective of mental health in youth and may be diminished during the process of acculturation (Stein, Gonzalez, Cupito, Kiang \& Supple, 2015). Interventions aimed at parents that explain the process of integration as an adaptive one may help them maintain familismo while at the same time allowing their children to integrate effectively into their new culture.

This decrease in family functioning may be especially difficult for girls. Although family functioning was not related to depression in girls, it was related to acculturative stress suggesting a potential indirect effect of family functioning for girls. Latino girls' acculturation may be accompanied by the deterioration of family cohesion and functioning due to the loss of Latino cultural values believed to discourage family conflict (Lorenzo-Blanco et. al, 2012). Because Latino adolescent females have greater sensitivity to interpersonal difficulties than their male counterparts, they may have more difficulty with family conflict, which places them at higher risk for depression (Lorenzo-Blanco et. al, 2012).

Our findings also highlight the importance of social support from various sources for these teenagers. Support from friends, family, teachers and school, the universe of relations for teenagers, were related to 
lower depressive symptoms in both girls and boys. These findings are not surprising, as the relation between social support and mental health is well understood, and has been previously examined among Latino teens in general (Gonzales, Fabrett \& Knight, 2009; Kuperminc, Wilkins, Roche \& Alvarez-Jimenez, 2009; Potochnick, Perreira \& Fuligni, 2012) and immigrant Latino youth (Blanco-Vega, Castro-Olivo \& Merrell, 2008). These findings do, however, suggest further avenues for addressing depressive symptoms in this population. These include strengthening the available support systems and finding new avenues to address the growing concerns over these teens' mental health.

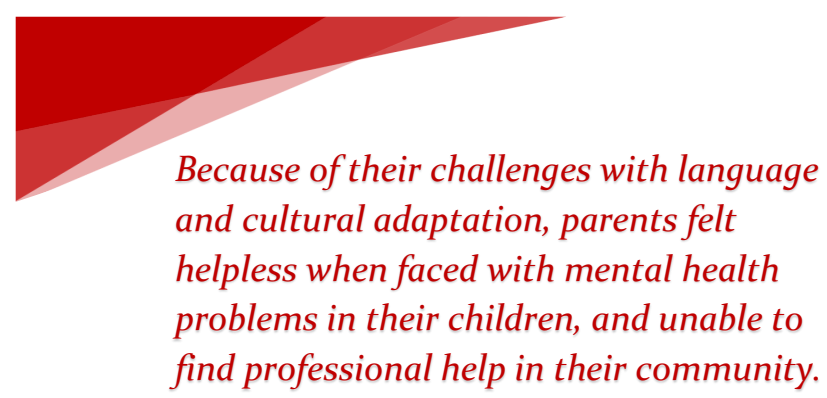

Strengthening teens' social support systems become especially important when considered in light of the main findings from the parent focus groups. Theme 4 from the focus groups presents both an explanation for the frustrations of parents and a course of action if interventions are to be tailored to parents or to changes in policies at the local, state, or national level. Because of their challenges with language and cultural adaptation, parents felt helpless when faced with mental health problems in their children, and unable to find professional help in their community. Although at several of the focus groups parents demonstrated interest in seeking help from mental health professionals, this was not always the case. Difficulties with getting Latino parents to seek mental health care for their children is well documented in the literature (McCord, Draucker \& Bigatti, 2018; Stafford, Aalsma, Bigatti, Oruche \& Draucker, 2019). This combination of reports from parents and extant literature suggest they are not likely to provide their teens with professional help unless it comes to them in the form of community-serving organizations that focus on mental health. Therefore, strengthening the available support systems, and finding new ones, is one way in which we can address the growing concerns over these teens' mental health; these support systems should include mental health professionals culturally competent to work with this population.

Our unique mixed methods approach, collecting quantitative data from adolescents and qualitative data from parents, was a strength of the study. It allowed for triangulation of data, and demonstrated that what happens within the home, what their parents perceive and how they behave, are important to understand adolescent depression and adaptation. The additional dimension of parental voice is often missing from studies of adolescents, and in the study reported here clarified many of the issues identified in the teens. It suggests a family-focused approach to interventions directed at this problem. Such an approach includes family therapy, parental training, and other approaches that consider the family unit as important when addressing problems in children and adolescents (Carr, 2014). There is evidence that this approach may be more effective than focusing on the individual child. A recent study of 
family-focused therapy for depression in children, with a sample that was $15 \%$ Latino, found that it resulted in better depression outcomes for the children and more satisfaction among families in all demographics included (Thompson, Sugar, Langer \& Asarnow, 2017).

The study presented here was a cross sectional, correlational, CBPR study, and as such, had inherent limitations. No conclusions can be drawn regarding causation among the variables. It is possible that depressive symptoms in the teens lead to problems in the variables we identified as predictors. It is also possible parental behaviors are caused by teen depression, and not the other way around. Although the triangulation of the teen's data with parental reports suggested explanations of one by the other, neither parents nor teens were privy to the others' data. Therefore, our conclusions are speculative and need to be confirmed in future research. Only studies that follow families over time, preferably upon arrival to the US or even earlier, would clarify causation. Of course, such studies are difficult with newlyimmigrated and low-income populations. Our survey instruments as well as our focus group questions sought to determine mostly what was happening, much more than why or how it was happening. This was purposeful, as it was a first step in understanding the needs of the community.

Furthermore, our study was focused mainly on the teens, their parents, and their immediate surroundings. The experiences these families may have with the larger community within Indiana and at the national level were not explored. Future research that explores the impact of national immigration policies and the increased discrimination occurring toward this population needs to be conducted. Research focused on parents suggests high psychological distress associated with immigration actions and national news (Roche, Vaquera, White \& Rivera, 2018). Convenience sampling most likely resulted in a biased sample, especially given that our community partner conducted the recruitment. However, in CBPR research it is the needs of the partner's constituents that are the focus of the collaboration, presenting both a limitation and strength of this type of research. These limitations related to CBPR, however, are counterbalanced by the importance of, and the ability to, reach a sample that seldom is represented in research.

In spite of these limitations, the quantitative data from the adolescents in the present study helps to understand factors associated with depression in Latino youth, and the qualitative data with parents clarified many of the dynamics between Latino teens and their parents during the difficult and uneven process of acculturation in these families. It suggests further avenues for research into this important problem and informs potential interventions at both the individual, family and community level. 


\section{REFERENCES}

Bellon-Saameno, J.A., Delgado-Sanchez, A., Luna del

Castillo, J.D. \& Lardelli-Claret, P.L., (1996)

Validez y fiabilidad del cuestionario de funcio'n familiar Apgar-familiar [Validity and reliability of the family Apgar family function test]. Atencion Primaria 18:289-296.

Blanco-Vega, C. O., Castro-Olivo, S.M., \& Merrell, K.W. (2008). Social-emotional needs of Latino immigrant adolescents: A sociocultural model for development and implementation of culturally specific interventions. Journal of Latinos and Education. 7(1), 43-61.

Boyatzis R. Transforming Qualitative Information: Thematic Analysis and Code Development. Thousand Oaks, CA: Sage; 1998.

Brown, A., Lopez, M. H. (2013). Mapping the Latino population, by state, county and city: Ranking Latino populations in the nation's metropolitan areas. Pew Research Center. Retrieved from:

http://www.pewhispanic.org/2013/o8/29/ivranking-latino-populations-in-the-nationsmetropolitan-areas/

Carr, A. (2014). The evidence base for family therapy and systemic interventions for child-focused problems. Journal of family therapy, 36(2), 107157.

Centers for Disease Control and Prevention (CDC). 1991-2015 High School Youth Risk Behavior Survey Data. Available at http://nccd.cdc.gov/youthonline/.
Céspedes, Y. M., \& Huey, S. J., Jr. (2008). Depression in Latino adolescents: A cultural discrepancy perspective. Cultural Diversity and Ethnic Minority Psychology, 14, 168-172.

Cox Jr., R. B., Zapata Roblyer, M., Merten, M. J., Shreffler, K. M., \& Schwerdtfeger, K. L. (2013). Do parent-child acculturation gaps affect early Latino alcohol use? A study of the probability and extent of use. Substance Abuse Treatment, Prevention, and Policy, 8(4).

Cupito, A., Stein, G. L., \& Gonzalez, L. M. (2014). Familial cultural values, gender, and psychological and academic risk and resilience. Journal of Child and Family Studies. DOI:10.1007/s10826-014-9967-7

Demaray, M., Malecki, C., \& Elliot, S. N. (2000). Child and adolescent social support scale. DeKalb, IL: Psychology Department, Nothem Illinois University.

Demaray, M. K., \& Malecki, C. K. (2003). Importance ratings of socially supportive behaviors by children and adolescents. School Psychology Review, 32, 108-131.

Dillon, F. R., De La Rosa, M., Ibañez, G. E. (2013). Acculturative stress and diminishing family cohesion among recent Latino immigrants. Journal of Immigrant Minority Health, 15, 484491.

Faul, F., Erdfelder, E., Buchner, A., \& Lang, A.-G. (2009). Statistical power analyses using $G^{*}$ Power 3.1: Tests for correlation and regression analyses. Behavior Research Methods, 41, 1149-116o. 
García, J. I. R., Manongdo, J. A., \& Ozechowski, T. J. (2014). Depression symptoms among Mexican American youth: Paternal parenting in the context of maternal parenting, economic stress, and youth gender. Cultural Diversity and Ethnic Minority Psychology, 20(1), 27.

Gilster, M. E. (2014). Neighborhood stressors, mastery, and depressive symptoms: Racial and ethnic differences in an ecological model of the stress process in Chicago. Journal of Urban Health: Bulletin of the New York Academy of Medicine, 91(4), 690- 706.

Gomez J.C. F., \& Ponce Rosas, E. R. (2010). A New Proposal of an Intepretation Scale for Family APGAR (spanish version). Atención Familiar, 17(4), 102-106.

Gonzales, N. A., Fabrett, F.C., \& Knight, G. P. (2009). Acculturation, enculturation, and the psychosocial adaptation of Latino youth. In F. Villarruel, G. Carlo, J. Grau, M. Azmitia, N. Cabrera, J. Chahin,(Eds.). Handbook of US Latino psychology: Developmental and community-based perspectives. (pp. 15134). Los Angeles, CA: Sage Publications.

Israel, B. A., Schulz, A. J., Parker, E. A., \& Becker, A. B. (1998). Review of community-based research: Assessing partnership approaches to improve public health. Annual Review of Public Health, 19, 173-202.

Kroenke, K., \& Spitzer, R. L. (2002). The PHQ-9: a new depression diagnostic and severity measure. Psychiatric Annals, 32(9), 509-515.
Kuperminc, G., Wilkins, N., Roche, C., \& AlvarezJimenez, A. (2009). Risk, resilience, and positive development among Latino youth. In F. Villarruel, G. Carlo, J. Grau, M. Azmitia , N. Cabrera, J. Chahin , (Eds.). Handbook of US Latino psychology: Developmental and community-based perspectives. (pp. 213-233). Los Angeles, CA: Sage Publications.

Lawton, K. E., \& Gerdes, A. C. (2014). Acculturation and Latino adolescent mental health: Integration of individual, environmental, and family influences. Clinical Child and Family Psychology Review, 17(4), 385-398.

Lorenzo-Blanco, E. I., Unger, J. B., BaezcondeGarbanati, L., Ritt-Olson, A., \& Soto, D. (2012). Acculturation, enculturation, and symptoms of depression in Hispanic youth: The roles of gender, Hispanic cultural values, and family functioning. Journal of Youth and Adolescence, 41(10), 1350-1365.

Lui, P. P. (2015). Intergenerational cultural conflict, mental health, and educational outcomes among Asian and Latino/a Americans:

Qualitative and meta-analytic review. Psychological Bulletin, 141(2), 404.

Malecki, C. K. \& Demaray, M. K. (2003). What type of support do they need? Investigating student adjustment as related to emotional, informational, appraisal, and instrumental support. School Psychology Quarterly, 18, 231-252.

McCord, A. L., Draucker, C. B., \& Bigatti, S. (2018). Cultural stressors and depressive symptoms in Latino/a adolescents: An integrative review. Journal of the 
American Psychiatric Nurses Association, 1078390318778885 .

Mena, F. J., Padilla, A. M., \& Maldonado, M. (1987). Acculturative stress and specific coping strategies among immigrant and later generation college students [Special issue]. Hispanic Journal of Behavioral Sciences, 9, 207-225.

Merz, E. L., Malcarne, V. L., Roesch, S. C., Riley, N., \& Sadler, G. R. (2011). A multigroup confirmatory factor analysis of the Patient Health Questionnaire-9 among English-and Spanish-speaking Latinas. Cultural Diversity and Ethnic Minority Psychology, 17(3), 309-316.

Miller, A. B., Esposito-Smythers, C., \& Leichtweis, R. N. (2015). Role of social support in adolescent suicidal ideation and suicide attempts. Journal of Adolescent Health, 56(3), 286-292.

Mojtabai, R., Olfson, M., \& Han, B. (2016). National trends in the prevalence and treatment of depression in adolescents and young adults.

Pediatrics, 138(6), http://dx.doi. org/10.1542/peds.2016-1878.

Pearlin, L.I. \& Schooler, C. (1978). The structure of coping. Journal of Health and Social Behavior 19(1), 2-21.

Pew Research Center (2014). Demographic Profile of Hispanics in Indiana. http://www.pewhispanic.org/states/state/in/ Pototochnick, S., Perreira, K.M., \& Fuligni, A. (2012). Fitting in: The roles of social acceptance and discrimination in shaping the daily psychological well-being of Latino youth. Social Science Quarterly, 93(1), 173-190.
Raffaelli, M. \& Ontai, L.L. (2004). Gender socialization in Latino/a families: Results from two retrospective studies. Sex Roles, 50 (5-6), 287-299.

Rini, C. K., Dunkel-Schetter, C., Wadhwa, P. D., \& Sandman, C. A. (1999). Psychological adaptation and birth outcomes: the role of personal resources, stress, and sociocultural context in pregnancy. Health Psychology, 18(4), 333.

Roche, C., \& Kuperminc, G. P. (2012). Acculturative stress and school belonging among Latino youth. Hispanic Journal of Behavioral Sciences, 34, 61-76.

Roche, K. M., Vaquera, E., White, R. M., \& Rivera, M. I. (2018). Impacts of immigration actions and news and the psychological distress of US Latino parents raising adolescents. Journal of Adolescent Health, 62(5), 525-531.

Rueger, S. Y., Malecki, C. K., Pyun, Y., Aycock, C., \& Coyle, S. (2016, in press). A metaanalytic review of the association between perceived social support and depression in childhood and adolescence. Psychological Bulletin. http://dx.doi.org/10.1037/buloooo058

SAMHSA. (2015). Behavioral health barometer: Indiana. Volume 4. Available at https://www.samhsa.gov/data/population-datansduh/reports?tab=33.

Scheier, M. F., Carver, C. S., Bridges, M. W. (1994). Distinguishing optimism from neuroticism (and trait anxiety, self-mastery, and self-esteem): A reevaluation of the Life Orientation Test. Journal of Personality and Social Psychology, 67(6), 1063-1078. 
Smilkstein, G. (1978). The family APGAR: A proposal for a family function test and its use by physicians. Journal of Family Practice 6(8).

Stafford, A. M., Aalsma, M. C., Bigatti, S. M., Oruche, U. M., \& Draucker, C. B. (2019). 4. Getting A Grip On My Depression: A Grounded Theory Explaining How Latina Adolescents Experience, Self-Manage, And Seek Treatment For Depressive Symptoms. Journal of Adolescent Health, 64(2), S2S3.

Stein, G. L., Gonzalez, L. M., Cupito, A. M., Kiang, L., \& Supple, A. J. (2015). The protective role of familism in the lives of Latino adolescents. Journal of Family Issues, 36(10), 1255-1273.

Stein, G. L., Gonzalez, L. M., \& Huq, N. (2012). Cultural stressors and the hopelessness model of depression in Latino adolescents. Journal of Youth and Adolescence, 41, 1339-1349.

Strange, R. (2013). Exploring Hoosier minority groups: Indiana's Hispanic population. INcontext: A
Publication of the Indiana Business Research Center at Indiana University's Kelley School of Business, July-August.

Thompson, M. C., Sugar, C. A., Langer, D. A., \& Asarnow, J. R. (2017). A randomized clinical trial comparing family-focused treatment and individual supportive therapy for depression in childhood and early adolescence. Journal of the American Academy of Child E Adolescent Psychiatry, 56(6), 515-523.

Wallerstein, N. \& Duran, B. (2010). Community-based participatory research contributions to intervention research: The intersection of science and practice to improve health equity. American Journal of Public Health, 100, 40-S46.

Watkins, D. C., Hudson, D. L., Caldwell, C.H., Siefert, K., \& Jackson, J. S. (2011). Discrimination, mastery, and depressive symptoms among African American men. Research on Social Work Practice, 21(3), 269277. 